\title{
NOTE-SUBMISSION FUNCTION FOR MOODLE QUIZ AND COLLECTING PEN-STROKE DATA
}

\author{
Yasuyuki Nakamura ${ }^{1}$ and Takahiro Nakahara ${ }^{2}$ \\ ${ }^{1}$ Graduate School of Informatics, Nagoya University, Nagoya, 464-8601, Japan \\ ${ }^{2}$ Sangensha LLC., Chitose, 066-0054, Japan
}

\begin{abstract}
Moodle, a Learning Management System (LMS), is a learning platform that provides flexible tool sets to support both blended learning and fully online courses. One of the most important features of LMS is online assessment, specifically, the computer-aided assessment (CAA) that allows evaluators to automatically and instantaneously assess students' answers as correct or incorrect. Currently, teachers can check students' answers but are not aware of the methodology used to obtain the solution. We have developed a module for Moodle through which students can submit their solutions along with related notes on their methodology and calculations by taking photographs or writing the notes directly onto devices, such as with a tablet and digital-pen. Therefore, teachers can view students' notes together with submitted answers to gain more in-depth understanding of the students' comprehension levels. Teachers can also insert feedbacks on the notes and return them to the students, who would consequently understand the subject better by referring to the notes. Furthermore, pen-stroke data on the note can be collected for further analytics.
\end{abstract}

\section{KEYWORDS}

Math e-Learning, Learning Analytics, Moodle, STACK

\section{INTRODUCTION}

One of the most important features of the e-Learning system is online assessment, specifically, the computer-aided assessment (CAA), as it allows evaluators to automatically and instantaneously assess students' answers as correct or incorrect. Common types of CAA questions are true-or-false and multiple-choice questions. There are other question types wherein students provide mathematical expressions as answers, determined by calculation; such a system is called a mathematics e-Learning system. STACK is one example of such systems. Teachers can also derive useful information from the students' answers, such as the kind of incorrect answers typically given by students and which students require tutoring. Even with these insights, however, the methodology associated with a student's solution remains unknown to the teacher. It is not adequate to solely evaluate answers when the student's level of comprehension remains unevaluated. This problem occurs in all the above-mentioned type of CAA questions.

To carry out CAA for e-Learning more effectively and efficiently, we developed a module for Moodle, a Learning Management System (LMS), in which students can submit calculation and methodology notes along with their answers. We call this function a 'note-submitting function'. This function was already developed as an input-type plug-in only for STACK. However, this function should be available for other question types on Moodle. Thus, we developed one-size-fits-all module not only for STACK but for any question type on Moodle.

\section{NOTE-SUBMITTING FUNCTION}

We implemented the note-submitting function by developing a question behaviour plug-in and a report-type plug-in for the quiz module of Moodle. The plug-ins have just been developed and have not yet been officially released. Those who wish to try the plug-ins can request a free copy of the code from the authors. 
The note-submitting function comprises two stages. The first stage is note-submission by the students who can submit notes on calculation and methodology used to obtain an answer. We specify two methods for students to submit notes: one is to submit the images of the corresponding notes that are suitable for mobile devices, and the other is to handwrite the notes directly onto the tablet-type device. When students submit images of a note using smartphones, they first write the calculations on a sheet of paper and then take their pictures (Figure 1(a)). As another option, if the students have tablet devices, they can submit their notes directly by writing them on their tablets with an electronic pen (Figure 1(b)).

The second stage is note-marking by the teachers. Teachers can view students' notes together with their submitted answers and can also insert comments onto the notes (Figure 1(c)), and students can subsequently review these comments (Figure 1(d)) for further learning.

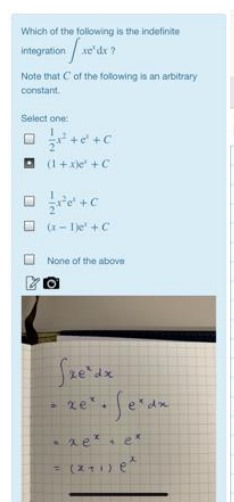

(a)

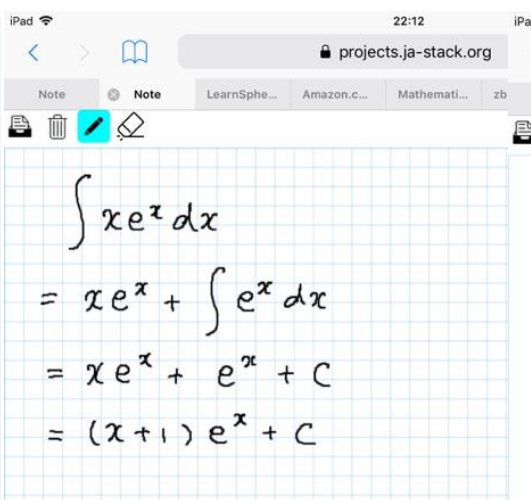

(b)

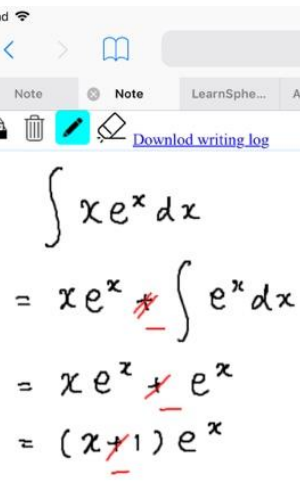

(c)

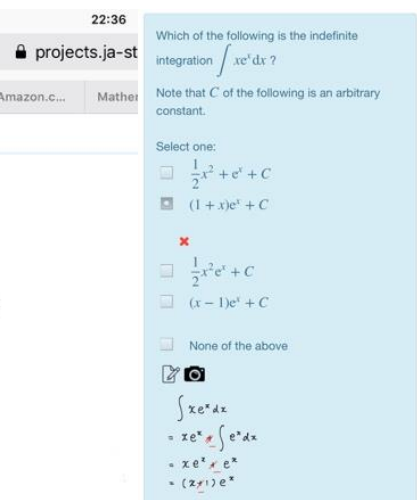

(d)

Figure 1. Note-submission by taking a picture (a) and by hand writing (b). Note-marking (c) and Review by Students (d)

\section{CONCLUSION}

We implemented the note-submission and note-marking functions for Moodle by developing a question behaviour plugin-in and a report-type plug-in for the Moodle quiz module. Students can submit notes by taking photographs of them or by writing the notes directly onto devices, such as with a tablet and digital pen. In addition, teachers can view students' notes together with submitted answers to gain in-depth understanding of the students' comprehension levels. Teachers can also insert comments on the notes and return them to the students, for better learning. Furthermore, pen-stroke data of students' notes can be collected and used for future learning analytics projects. How frequently students rewrite their notes by using the eraser could be related to the students' comprehension level. OCR could be applied and automatic computer-based assessment might be possible but this challenging project is the subject for future research.

\section{ACKNOWLEDGEMENT}

This work was supported by JSPS KAKENHI Grant Numbers 17K18625.

\section{REFERENCES}

Nakamura, Y. andNakahara, T., 2017. Developmentof a note-submittingfunction for themathematics e-Learningsystem STACK. Presented at The 12th Workshop on Mathematical User Interfaces.Edinburgh, U.K.

Sangwin, C., 2013. Computer Aided Assessment of Mathematics. Oxford University Press. 